\title{
Observations on the Linear Order of Program Code Reading Patterns in Programmers with Dyslexia
}

\author{
Ian McChesney \\ School of Computing \\ Ulster University \\ Newtownabbey, UK \\ ir.mcchesney@ulster.ac.uk
}

\author{
Raymond Bond \\ School of Computing \\ Ulster University \\ Newtownabbey, UK \\ rb.bond@ulster.ac.uk
}

\begin{abstract}
The software engineering industry is increasingly aware of the role and value of neurodiverse engineers within the workforce. One motivation is the alignment between skills needed for software development and the processing strengths of individuals with autistic spectrum conditions. One aspect of neurodiversity is dyslexia, typically presenting in individuals through a range of reading deficiencies. In this paper we build on recent work which has sought to investigate if programmers with dyslexia read program code in a way which is different from programmers without dyslexia. The particular focus of this analysis is the nature of saccadic movement and patterns of linearity when reading code. A study is presented in which the eye gaze of 28 programmers (14 with dyslexia and 14 without) was recorded using an eye tracking device while reading and understanding three on-screen Java programs. Using insights from the wider dyslexia literature, hypotheses are formulated to reflect the expected saccadic gaze behaviour of programmers with dyslexia. A range of existing metrics for linearity of program reading are adapted and used for statistical analysis of the data. Results are consistent with recent work elsewhere and indicate that programmers with dyslexia do not exhibit patterns of linearity significantly different from the control group. Non-linear gaze is shown to be approximately $40 \%$ of all saccadic movement. Some preliminary insights are offered based on the data available, suggesting that the extent of non-linear reading when comprehending program code might complement the processing and problem solving style of the programmer with dyslexia.
\end{abstract}

\section{CCS CONCEPTS}

-Software and its engineering $\rightarrow$ Maintaining software; $\bullet$ Humancentered computing $\rightarrow$ Accessibility design and evaluation methods.

\section{KEYWORDS}

Computer programming, program comprehension, eye tracking, software maintenance, dyslexia, empirical study.

Permission to make digital or hard copies of all or part of this work for personal or classroom use is granted without fee provided that copies are not made or distributed for profit or commercial advantage and that copies bear this notice and the full citation on the first page. Copyrights for components of this work owned by others than ACM must be honored. Abstracting with credit is permitted. To copy otherwise, or republish, to post on servers or to redistribute to lists, requires prior specific permission and/or a fee. Request permissions from permissions@acm.org.

EASE 2020, April 15-17, 2020, Trondheim, Norway

(C) 2020 Association for Computing Machinery.

ACM ISBN 978-1-4503-7731-7/20/04 . \$ \$15.00

https://doi.org/10.1145/3383219.3383228
ACM Reference Format:

Ian McChesney and Raymond Bond. 2020. Observations on the Linear Order of Program Code Reading Patterns in Programmers with Dyslexia. In Evaluation and Assessment in Software Engineering (EASE 2020), April 15-17, 2020, Trondheim, Norway. ACM, New York, NY, USA, 9 pages. https: //doi.org/10.1145/3383219.3383228

\section{INTRODUCTION}

There is an increasing awareness in the fields of computing and software engineering of the value of neurodiversity in the workplace. Dalton [10] argues that research and practice in HCI (HumanComputer Interaction) needs to realign to take account of the characteristics and cognitive strengths of the neurodiverse community - individuals who can be considered as exhibiting, for example, dyslexia, dyscalculia, dyspraxia, autistic spectrum disorder or Asperger's syndrome. Morris et al. [22] report findings from a survey of neurodiverse professional programmers investigating challenges and opportunities in the workplace. They identify challenges such as knowing when it is appropriate to depart from rigid processes in the workplace and opportunities such as efficacy in tasks reliant upon compliance with good programming style and practice. Anabi et al. [2] note the trend in some large software engineering companies to target recruitment amongst the neurodiverse community. The intention of such companies is to align skills required in software development with the strengths of technologists who have autism spectrum conditions. For example, individuals with dyslexia may have talent for visual processing and spatial reasoning [26], [15].

In the field of program comprehension, recent work has investigated the code reading behaviour of programmers with dyslexia, seeking to establish if such programmers read and ultimately understand code in ways which are different from programmers without dyslexia [21]. Using eye tracking data, initial results from this work suggest that such programmers do not exhibit code reading patterns that would be expected of dyslexic readers in natural text contexts. The analysis in [21] focused mainly on metrics related to fixations on key program features as the mechanism by which to distinguish behaviour between programmers with and without dyslexia. The analysis reported in this paper, using the dataset from [21], concentrates on saccade-related metrics to explore possible differences in gaze behaviour. In particular, drawing upon the work of Busjahn et al. [6], the present analysis seeks to establish if the linearity of code reading is different between programmers with and without dyslexia.

For programmers who do not have dyslexia, the observations in this paper relating to linearity in code reading are consistent 
with [6]. For programmers with dyslexia, while recognizing that dyslexia is a multi-faceted condition, the analysis reported here is broadly consistent with the findings in [21] suggesting that this aspect of neurodiversity may not actually present any detrimental factors in code reading ability.

\section{RELATED WORK}

In their review of the psychology of programming, Blackwell et al. [5] identify program comprehension as an established and active area of enquiry. It is fundamental to the task of software maintenance, especially when working with code which has been written by someone else. In recent years eye tracking has become a useful empirical technique for the study of program comprehension, enabling the researcher to record and analyze the programmer's gaze when reading program code. In Obaidellah et al's. mapping study on the usage of eye tracking in computer programming [23], program comprehension is the topic which has received most attention by the research community, followed by topics such as debugging, collaborative programming and requirements traceability.

The Eye Movements in Programming series of workshops [1] has provided an important forum for the exchange of ideas in this relatively new field. It has helped to, for example, (a) define a tentative vocabulary for the fine grained description of observable gaze patterns in reading program code, articulating patterns such as Flicking, JumpControl, LinearHorizontal, LinearVertical, RetraceReference and Scan [3], (b) understand differences in gaze behaviour between novice and expert programmers [3],[6] and (c) disseminate analytical tools for visualization of eye gaze in a programming context [7].

The gaze metrics used in eye tracking in software engineering can be broadly considered as metrics derived from fixations, saccades or scan paths [27]. A fixation is where gazed is stablized on a location for typically $200-300 \mathrm{~ms}$ [18] and is a strong indicator of attention and cognitive processing. A saccade is an eye movement traversing from one fixation to another, with a duration in the range $30-80 \mathrm{~ms}$ [18] and is typically associated with searching or navigating the visual information space. A scan path is an ordered sequence of fixations, typically associated with some finite task of reading, understanding or searching. In the study of program comprehension, fixation related measures are the dominant metrics used in research studies due to their link with cognitive load and attention. Sharafi et al. [27] note that only a small number of studies in program comprehension have used saccades as metrics.

The code artefact under study in eye tracking experiments is typically a program or program segment which can be viewed on a computer screen. In this way, reliable fixation, saccade and scan path metrics can be generated. This does not always reflect professional code comprehension settings. To address this limitation, Sharif et al. [28] describe iTrace, an Eclipse plug-in, which interfaces with an eye tracker and can synchronize gaze data with other edit and workflow operations logged through the IDE. This approach helps to enrich basic gaze data for use in semantic analysis of program interaction. This is especially useful where the artefact under study is not a static code segment but is a live code file linked dynamically with other artefacts such as class definitions in the IDE. In this way, gaze behaviour in an industrial context can be gathered.
Jbara and Feitelson [19] used eye tracking to show that programmers tasked with reading and understanding "regular code" (program code which includes repetitions of the same basic pattern) take advantage of the repeated patterns to improve efficiency of program comprehension. This in turns leads to the necessity of reinterpreting complexity metrics for regular code, such as McCabe's Cyclomatic Complexity and LOC counts, to take account of these comprehension efficiencies.

Eye tracking technology has an established presence in the study of reading natural text, especially in the context of developmental psychology and models of reading [24]. It has also enabled a sophisticated analysis of reading disability and has helped to delineate some expected gaze behaviours of the dyslexic reader. Though not conclusive, many studies point to dyslexic readers exhibiting longer fixations, shorter saccades and more regressions. Other patterns observed are that increased word length leads to significantly increased fixation duration and that dyslexic readers shows less tendency to scan or skip words when reading [4].

The work described in [21] uses this body of work as a basis for investigating the reading behaviour of programmers with dyslexia. It also acknowledges that dyslexia is a multi-faceted condition and that the different types of dyslexia, such as position dyslexia, attentional dyslexia, letter identity dyslexia, and phonological dyslexia [13] are not accounted for at this stage of investigation. Nevertheless, the results reported show that in general terms programmers with dyslexia do not exhibit radically different gaze behaviours compared to programmers without dyslexia and they tend not to follow the expected gaze behaviours of dyslexic readers. Potential issues regarding the impact of code crowding on programmers with dyslexia require further investigation.

McChesney and Bond's study [21] has only a limited analysis of the saccadic aspect of gaze behaviour. In this paper, we use extensively the analysis framework presented in [6] which provides metrics for investigating the linearity of gaze behaviour when reading program code. Busjahn et al. used this to better understand the mix of linear and non-linear gaze patterns and to establish differences in linear order between novice and expert programmers. Relevant aspects of their approach are introduced in the following sections.

\section{METHOD}

\subsection{Study Design}

Data collection involved 28 participants, consisting of a study group of 14 programmers with dyslexia (mean programming experience $=3.32$ years, $\mathrm{SD}=2.44$ ) and a control group of 14 programmers without dyslexia (mean programming experience $=2.89$ years, $\mathrm{SD}=$ 1.47). No other aspects of neurodiversity were elicited or recorded from the participants. For all participants, normal reading mode was left-to-right. Recordings took place in a private university study room with natural lighting. For each recording, only the participant and an investigator were present. Subjects were presented with three on screen Java programs, adapted from the EMIP'14 workshop [3] - see Figures 1-3. The programs were displayed on $56 \mathrm{~cm}(22$ inch) Flat Panel Monitor operating at a resolution of $1280 \times 800$ at $60 \mathrm{~Hz}$. The font size at which text and program code was displayed was $5 / 16$ inch (22.5 point size). The selected programs included 
a mix of sequence, selection and iteration constructs. They were small enough to fit on a single screen while maximizing font size and were sufficiently complex to engage the participant for a period of time to acquire meaningful gaze data.

Eye gaze in each session was recorded using a Tobii X60 Eye Tracker with a typical accuracy of 0.5 degrees and spatial resolution of 0.2 degrees. Participants were seated in front of the Tobii recorder at a distance of approximately $50 \mathrm{~cm}$, with the distance from the Tobii lens to eye approximately $70 \mathrm{~cm}$. Random error arising from the tracker accuracy limitations was mitigated through calibration for each participant, maximizing the size of areas of interest and the collection of a large number of gaze points per participant.

For each program, the participant was asked to read the code and then describe to the investigator the program's purpose. In addition, participants completed a short profiling questionnaire, capturing data such as preferred programming language, programming experience and a self assessment of fatigue. On this questionnaire participants also indicated if they had dyslexia and were asked to provide a self-assessment rating of their dyslexia as mild, moderate or severe. For each participant, the investigators also completed a comprehension assessment on a scale of 1-10 (1 = no understanding through 10 = full understanding). The study was approved by the appropriate University research ethics committee.

For this paper, our Research Question was "Do programmers with dyslexia have different linear and non-linear reading behaviours when reading code compared to programmers without dyslexia?" Linearity was in part addressed by [21] through consideration of regression as measured by the visit count metric. In this analysis, however, we use saccade data to more fully explore linearity in terms of the following hypotheses:

Hypothesis 1

Programmers with dyslexia have shorter saccades when reading program code than the control group.

\section{Hypothesis 2}

For programmers with dyslexia, the proportion of all saccades which are linear saccades is less than the proportion associated with the control group.

Hypothesis 1 is derived from work presented by, among others, Bellocchi et al. [4] and Hawelka et al. [17]. This work has established that, when reading natural text, at word, pseudoword or sentence level, dyslexic eye movements are characterized by shorter saccades, longer fixations and more regressions.

Hypothesis 2 is concerned with the proportion of linear to nonlinear saccades when reading program code. Busjahn et al. [6] showed that novice programmers exhibited greater linear behaviour than expert programmers, suggesting that expert programmers are able to scan key program features in order to formulate the purpose of a program. In this context, it can be assumed that linear order is associated with a more methodical approach to code reading. The dyslexia literature has established that, when reading natural text, dyslexic readers exhibit a smaller number of words that receive a single fixation or that are skipped, and a greater number of words with multiple fixations [4]; [11]. This is associated with a more "uncertain" reading approach, to compensate for the visual and
Table 1: Linear and non-linear saccade metrics

\begin{tabular}{ll}
\hline Linear & \\
Horizontal Later (HL) & $\mathrm{FS} \wedge(\mathrm{L} x(\mathrm{t})-\mathrm{L} x(\mathrm{~s})=0)$ \\
Horizontal Strict (HS) & $\mathrm{HL} \wedge(\mathrm{W} y(\mathrm{t})-\mathrm{W} y(\mathrm{~s})=1)$ \\
Vertical Next (VN) & $\mathrm{DS} \wedge(\mathrm{L} x(\mathrm{t})-\mathrm{L} x(\mathrm{~s})=1)$ \\
Vertical Strict (VS) & $\mathrm{VN} \wedge(\mathrm{W} y(\mathrm{t})-\mathrm{W} y(\mathrm{~s})=1)$ \\
Vertical Later (VL) & $\mathrm{DS} \wedge(\mathrm{L} x(\mathrm{t})-\mathrm{L} x(\mathrm{~s})>1)$ \\
\hline Non-linear & \\
Horizontal Earlier (HE) & $\mathrm{BS} \wedge(\mathrm{L} x(\mathrm{t})-\mathrm{L} x(\mathrm{~s})=0)$ \\
Vertical Previous (VP) & $\mathrm{US} \wedge(\mathrm{L} x(\mathrm{t})-\mathrm{L} x(\mathrm{~s})=-1)$ \\
Vertical Earlier (VE) & $\mathrm{US} \wedge(\mathrm{L} x(\mathrm{t})-\mathrm{L} x(\mathrm{~s})<-1)$ \\
\hline
\end{tabular}

lexical deficiencies in text processing. This uncertain approach can manifest as scanning and regression. Regression occurs necessarily, for example, when reading a program loop or method call according to the program's control flow. Regression can also be a case of simply referring back as a reminder to earlier parts of the program text. Scanning can involve regression but also involves forward saccades such as jumping ahead, horizontally or vertically, to gather information about later parts of the program. Reflecting the more uncertain reading style expected from dyslexic readers, hypothesis 2 states that the proportion of linear reading is less for programmers with dyslexia compared to the control group.

In addition to testing the hypotheses, exploratory data analysis was also performed to establish any other linear patterns associated with the two groups. This included a line-by-line analysis of gaze behaviour across the two groups and a consideration of any differences in so called story order gaze or execution order gaze.

\subsection{Eye Gaze Metrics}

Busjahn et al. [6] present a set of gaze-based metrics for describing the linearity of code reading behaviour. With some minor adjustment and extension, we adopted these measures as the basis for this analysis. Each saccade in the dataset was labelled as a forward (FS), backward (BS), downward (DS) or upward saccade (US), according to its absolute saccadic direction [18] (Figure 4). Saccades were then labelled with reference to program areas of interest, namely lines of code and program words. These areas of interest are designated according to (a) the program line of code index ( $\mathrm{L} x, 1<=x<=\mathrm{n}, \mathrm{n}$ $=$ number of lines of code) and (b) program words index (operators and operands) (Wy, $1<=y<=\mathrm{m}, \mathrm{m}=$ total number of words in the program ordered top to bottom, left to right). Saccades can then be categorized as linear or non-linear with reference to their source (s) and target ( $\mathrm{t}$ ) as shown in Table 1 (see also Figure 5).

We also adopted from [6] the notion of story order and execution order reading patterns. Story order is where the programmer reads the code in a linear manner according to its top to bottom, left to right ordering on the screen, as when reading natural text. Execution order is where the programmer reads the code according to the control flow of the program. For example, when reading an if statement, the programmer may have a conditional value in mind and use this to guide her gaze to the true or false branch of the statement accordingly. Furthermore, story order or execution order may not be limited to describing one pass through the code but might 


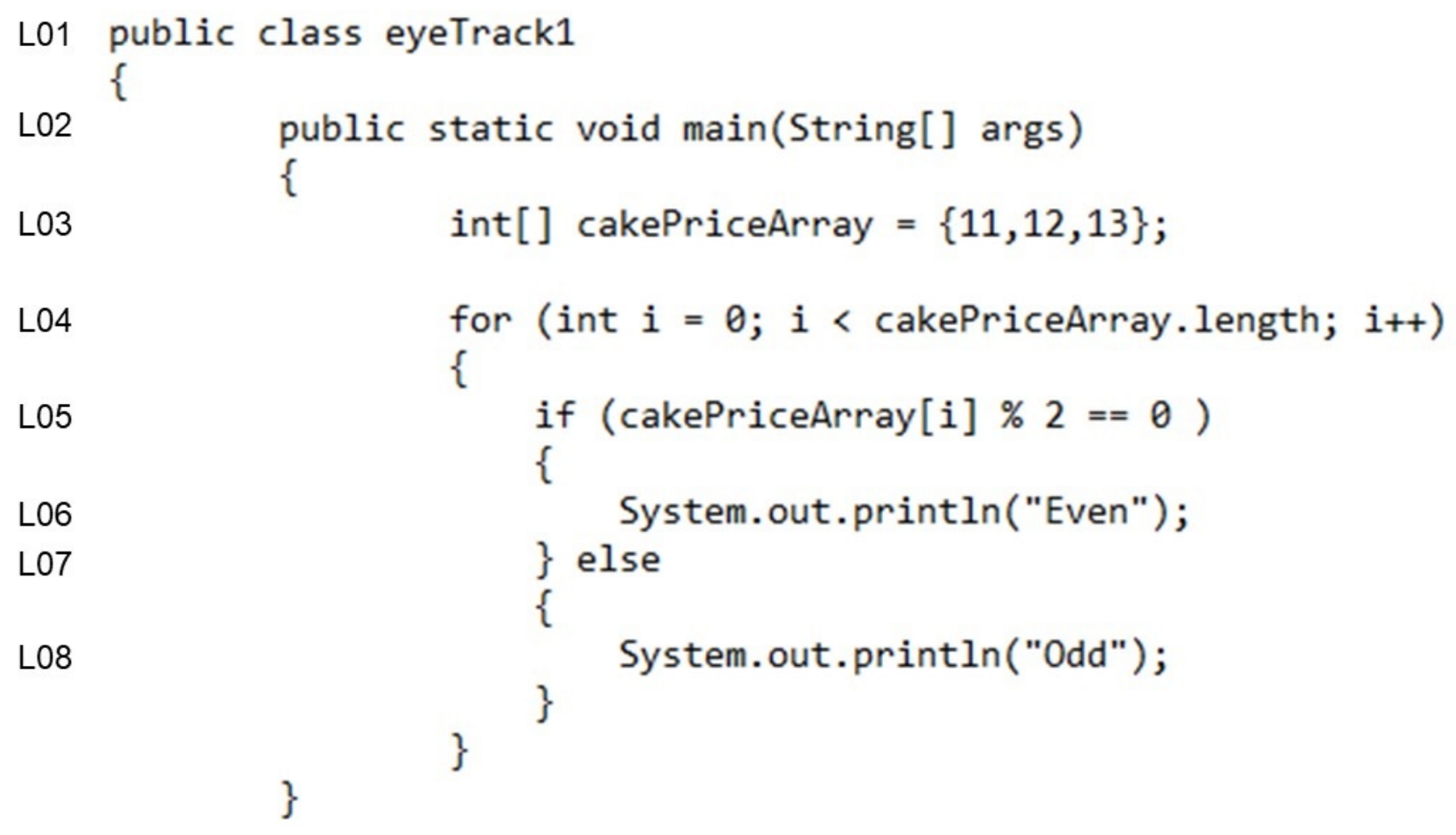

Figure 1: Program 1

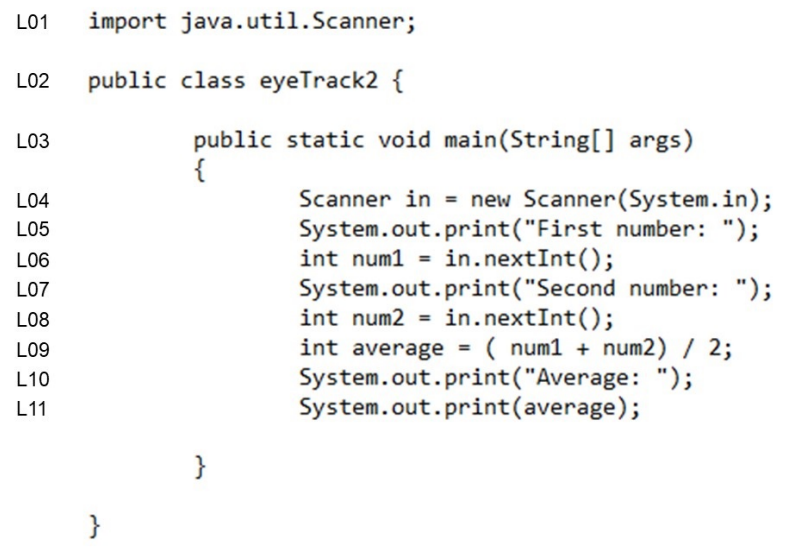

Figure 2: Program 2

include repetitions of all or part of the program. When program lines of code or program features are labelled as areas of interest as above, then story and execution order can be represented as a sequence of strings accordingly. See for example, figure 5 where, if we assume line 4 is conditional upon line 3, then story order is 12345 and an execution order, for a given input space, might

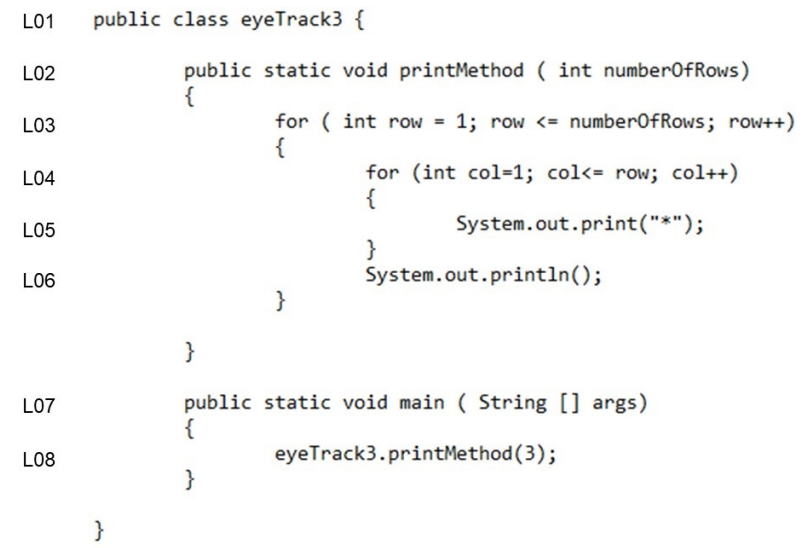

Figure 3: Program 3

be 1235. Christino et al. [9] describe how the Needleman-Wunsch (N-W) sequence alignment algorithm can be used to compare gaze patterns from eye gaze recordings and the study in [6] uses this for describing patterns of code reading. 


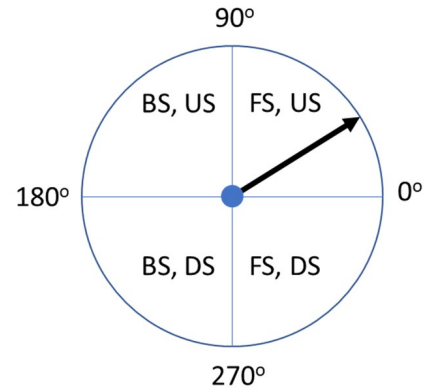

Figure 4: Absolute saccadic direction. BS - backward saccade, DS - downward saccade, FS - forward saccade, US - upward saccade

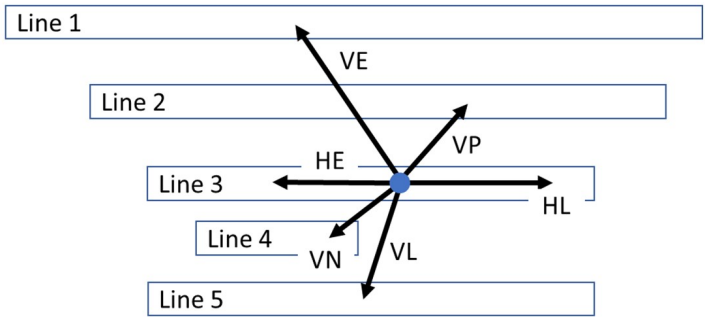

Figure 5: Categories of linear and non-linear gaze. HE - Horizontal Earlier, HL - Horizontal Later, VE - Vertical Earlier, VL - Vertical Later, VN - Vertical Next, VP - Vertical Previous

\section{ANALYSIS AND RESULTS}

To investigate the hypotheses, we compared the above metrics across groups - dyslexia group and control group. For hypothesis 1 we analyzed saccade amplitude at program level and line level. For hypothesis 2 we counted HL, VN and VL and added these to give a count of linear saccades; we counted HE, VP and VE and added these to give a count of non-linear saccades. In this way we could quantify the proportion (percent) of linear versus non-linear saccades at program level and per line of code. Exploratory analysis also used these metrics in addition to $\mathrm{N}-\mathrm{W}$ similarity scores for story order and execution order reading.

\subsection{Hypothesis 1 - Saccade Amplitude}

The hypothesis was tested using a one-tailed independent samples ttest for equality of means at a significance level $p$ of 0.05 . Inequality of variances in the sample values was accounted for using Levene's Test. Any adjustment to the degrees of freedom arising from this is reported as $\mathrm{t}(\mathrm{df})$ in the following tables and presentation of results. Where appropriate, effect size is reported using Cohen's $d$ metric.

Saccade magnitude is expressed in visual degrees. When average values were taken at the program level (Table 2), the dyslexia group
Table 2: Saccade Amplitude (degrees)

\begin{tabular}{cccccccc}
\hline saccade amplitude & \multicolumn{2}{c}{ dyslexia } & \multicolumn{2}{c}{ control } \\
\hline Program & mean & SD & mean & SD & t(26) & p \\
\hline 1 & 3.18 & 0.58 & 3.28 & 0.58 & -0.48 & .317 \\
2 & 3.09 & 0.71 & 3.27 & 0.59 & -0.72 & .240 \\
3 & 3.23 & 0.61 & 3.33 & 0.61 & -0.44 & .333 \\
\hline
\end{tabular}

Table 3: Proportion (\%) of saccades which are linear

\begin{tabular}{ccccccc}
\hline linear\% & \multicolumn{2}{c}{ dyslexia } & \multicolumn{2}{c}{ control } & & \\
\hline program & mean & SD & mean & SD & $\mathrm{t}(26)$ & $\mathrm{p}$ \\
\hline 1 & 52.71 & 12.80 & 54.07 & 8.89 & -0.33 & .374 \\
2 & 56.64 & 3.34 & 59.43 & 5.56 & $-1.61(21)$ & .062 \\
3 & 57.36 & 6.46 & 58.86 & 5.93 & -0.640 & .264 \\
\hline
\end{tabular}

show consistently shorter saccades than the control group. While in keeping with the hypothesis, the differences are not statistically significant.

When saccade amplitude was analyzed per line of code across the two groups, the only significant difference to emerge was in program 2, line 5 (dyslexia mean $=3.05, \mathrm{SD}=0.89$; control mean $=$ $3.76, \mathrm{SD}=0.65 ; \mathrm{t}=-2.43, \mathrm{p}=.011, d=-0.91)$. Taking all of the data into account, there is no strong evidence to suggest that programmers with dyslexia have shorter saccades than the control group. We observe that the saccade amplitude figures are consistent with those of expert programmers (3.12 degrees) as reported in [6].

\subsection{Hypothesis 2 - Linear Order}

4.2.1 Proportion of linear versus non-linear saccades at program level (all lines). For each participant, we computed across all lines of code the total number of linear saccades and calculated their proportion with respect to all of the participant's saccades on the line of code. As previously noted, a linear saccade is any saccade categorized as HL, VN or VL. Saccades which do not have a source and target on a line of code are not counted, so that the saccades counted are those directly linked with the code reading process. This is also consistent with the approach described in [6].

We defined linear\% as the proportion of linear saccades over all linear and non-linear saccades when reading the program code. Comparing linear\% across the two groups at the program level, we have data as shown in Table 3 . Hypothesis 2 predicts the dyslexia group to have less linearity in their reading pattern. Table 3 suggests less linearity, and though the difference in values is not statistically significant, we note program 2 exhibits this trait more so than the other two programs.

Comparing with [6], we observe that our control group figures are slightly lower than their "expert" linearity value of $60 \%$.

4.2.2 Proportion of linear versus non-linear saccades at line level. When considering linear\% per line, the linear saccade metrics of interest are again HL, VN and VL. VN and VL saccades are counted against the target line (landing point) of the saccade. Similarly HE, VP and VE are counted as non-linear saccades for the target line. 
Table 4: Program 2 - Differences in linear\%

\begin{tabular}{cccccccc}
\hline linear\% & \multicolumn{2}{c}{ dyslexia } & \multicolumn{2}{c}{ control } & & & \\
\hline line & mean & SD & mean & SD & $\mathrm{t}(26)$ & $\mathrm{p}$ & $d$ \\
\hline 1 & 35.64 & 35.05 & 56.90 & 30.51 & -1.57 & .065 & -0.65 \\
2 & 46.79 & 35.97 & 49.03 & 35.87 & -0.16 & .438 & -0.06 \\
3 & 42.33 & 30.95 & 71.51 & 31.77 & -2.31 & .015 & -0.93 \\
4 & 37.07 & 30.83 & 41.42 & 21.53 & -0.39 & .351 & -0.16 \\
5 & 54.32 & 10.94 & 59.07 & 21.42 & -0.74 & .233 & -0.28 \\
6 & 55.55 & 9.72 & 55.36 & 13.43 & 0.04 & .484 & 0.02 \\
7 & 57.60 & 10.12 & 56.07 & 13.80 & 0.33 & .372 & 0.13 \\
8 & 58.25 & 14.61 & 61.55 & 14.47 & -0.60 & .277 & -0.23 \\
9 & 55.04 & 19.75 & 69.98 & 16.68 & -2.16 & .020 & -0.82 \\
10 & 63.31 & 16.25 & 58.03 & 21.01 & 0.74 & .232 & 0.28 \\
11 & 78.63 & 10.46 & 74.72 & 15.65 & 0.76 & .228 & 0.29 \\
\hline
\end{tabular}

The experimental data also contains saccades landing on a line but the source is a region on the screen not delineated as a line of code, such as white space between a line of code and the edges of the program screen. For the purpose of describing the linearity of code reading, these saccades are counted against the target line.

When the linear\% data is considered on a per line basis, the Shapiro-Wilks test of normality typically showed a normal distribution, though for some "short" lines the data did have a non-normal distribution (e.g. program 1, line 7). When counts of linear and non-linear saccades are considered, the data consistently shows a non-normal distribution. Given the normality for the proportion values, we used the independent t-test when comparing linear\% across the two groups. For all lines of code in programs 1 and 3 there was no significant difference. For program 2, we saw significant differences for lines 3 and 9. This is shown in Table 4, with linear\% values for all lines of code to provide context. (There were similar findings when using the Mann-Whitney $U$ test).

We observed that for some lines, typically at the beginning of the program, there is a low value for linear\% reading. This is to be expected for these lines of code since, as reading progresses, scanning to lines at the beginning of the program will count as nonlinear saccades (regressions) as part of the program comprehension process.

To see if saccades are more "scattered" for one group compared to the other, we also analyzed VL and VE (the metrics which capture the largest saccades in terms of program coverage) and HS and VS (the metrics which capture the smallest linear saccades). At the program level, there was no significant difference for program 1 nor program 3. For program 2, there was no significant difference except for the metric VS\% (dyslexia mean $=0.34$, SD $=0.41$; control mean $=0.82, \mathrm{SD}=0.85 ; \mathrm{t}=-1.94, \mathrm{p}=.031, d=-0.72$ ). Similar analysis was conducted per line across the two groups. Table 5 shows the lines of code and metrics which exhibited a significant difference.

Regarding the patterns observed from this data, by counting the number of lines for which the dyslexia group showed a greater or lesser value than the control group (Table 6), we observed a consistent pattern of the dyslexia group exhibiting a greater proportion of jumping, linear and non-linear, across multiple lines, though the data did not show statistical significance. For program 1 and program 2, proportions for the shortest horizontal linear saccades (HS\%) were similar for both groups; for program 3 they were less for the dyslexia group. The shortest vertical linear saccade (VS\%) data indicated the dyslexia group showing less such gaze behaviour for program 1 and program 2 but similar gaze behaviour for program 3.

As such, the tendency is for the dyslexia group to exhibit more jumping (VL and VE), both linear and non-linear, though there are limited instances of this being statistically significant. Nevertheless, as a tentative observation, the data is suggesting gaze behaviour in keeping with hypothesis 2 - programmers with dyslexia exhibiting a proportion of linear saccades less than the proportion associated with the control group or, in other words, more non-linear gaze behaviour.

\section{EXPLORATORY ANALYSIS}

\subsection{Comparing linear gaze across lines}

In this part of the analysis we did not separate the data into dyslexia and control groups. Instead our purpose was to identify any patterns in the proportion of linear versus non-linear saccades across lines of code in each program. For this we conducted One-way ANOVA tests on linear\% between lines. This was to establish how individual lines of code were associated with differences in linearity of gaze patterns. For program 1, the line pairs which showed significant differences in the proportion of linear gaze are shown in Table 7. For line 8 this is to be expected, as it is the last line in the program and will receive very few non-linear saccades. For line 2 , as the signature of the method, this would suggest it is playing a limited role in the programmer's comprehension of the program given the low non-linear gaze associated with it.

In program 2, line 11 received significantly more linear\% gaze than all of the other lines - again, this is a function of its location as the last line in the program. In program 3 the last line has a significantly more linear\% and line 1 shows significantly more nonlinear\% gaze than the other lines in the program.

The outcome of this analysis confirms what would be expected in that the distribution of linear and non-linear gaze is not uniform across the lines of code but is spread according to the location of a line towards either the beginning or end of the program.

\subsection{Story versus Execution Order}

Busjahn et al. [6] describe how the Needleman-Wunsch algorithm (with its origins in bioinformatics) can be used as a way of comparing actual gaze to either the story order or execution order of program execution. In this part of our analysis we investigated if there were any differences in this aspect of gaze between the dyslexia and control groups.

Cristino et al. [9] describe an adaption and implementation of the Needleman-Wunsch algorithm for use in comparing similarities in eye gaze patterns. Busjahn et al. [6] showed how this could be used for comparing the eye gaze of a programmer against an idealized or "model" gaze pattern for reading a particular program. The model gaze pattern can take one of two forms. Story order is the case where gaze follows the reading order of the code as it appears on screen, reading top to bottom, left to right. Execution order is where the gaze follows the control flow of the program; this will have 
Table 5: Differences in saccade "scatter"

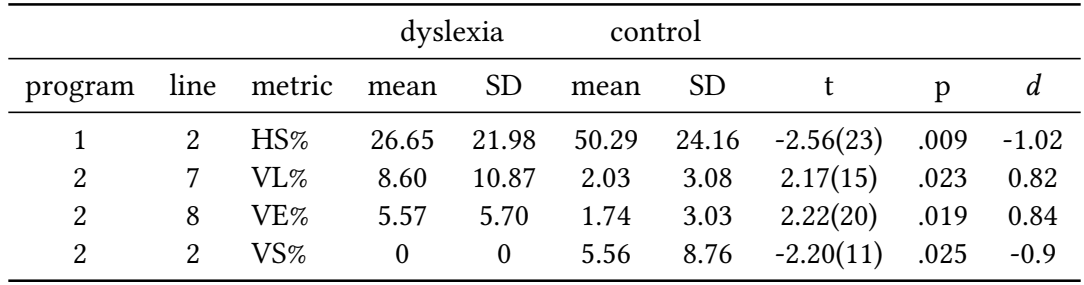

Table 6: Summary of saccade "scatter" across the programs (dyslexia group $[>|=|<]$ control group)

\begin{tabular}{ccccc}
\hline program & VL\% & VE\% & HS\% & VS\% \\
\hline 1 & $>$ & $>$ & $=$ & $<$ \\
2 & $>$ & $>$ & $=$ & $<$ \\
3 & $>$ & $>$ & $<$ & $=$ \\
\hline
\end{tabular}

significant elements of story order but will also include non-linear components when, for example, the programmer is following a method call or when reading according to the control flow of a loop structure. For example, using line numbers, the story order for program 1 in Figure 1 is 12345678 and the execution order is 12345784564578 .

Using saccade data we were able to construct the gaze sequence for each participant and describe this using line numbers as above. For each participant, their gaze sequence was compared to the program's model story order and model execution order. As noted in [6], during comprehension a programmer will typically read a program more than once. As such, the length of the gaze sequence will be longer than the model sequence. When comparing sequence strings, therefore, the model sequence is repeated, for each specific comparison, sufficient times to be equal to or greater than the participant's gaze.

The Needleman-Wunsch algorithm required two parameters to be configured. The substitution matrix is a $n \times n$ matrix ( $n$ is the number of lines in the model sequence) which specifies the scoring to be used when there is a match or mismatch in the string sequence comparison. For example, all the diagonals on the matrix specify the score when a line number in the two gaze sequences match. In our case we set this value to $2\left(\mathrm{~S}_{i j}=2, i=j\right)$. Other pairs in the matrix can be used to score the presence of a mismatch, if

Table 7: Program 1 - Significant differences in linear\% across lines

\begin{tabular}{cccccccc}
\hline linear\% & \multicolumn{2}{c}{ line (a) } & \multicolumn{5}{c}{ line $(\mathrm{b})$} \\
\hline line (a) & mean & SD & line $(\mathrm{b})$ & mean & SD & $\mathrm{p}(2 \mathrm{t})$ & $d$ \\
\hline 2 & 67.98 & 21.31 & 1 & 40.77 & 26.68 & .008 & 1.13 \\
& & & 3 & 43.33 & 24.19 & .012 & 1.44 \\
8 & 75.95 & \multirow{2}{*}{30.99} & 1 & 40.77 & 26.68 & .010 & 1.22 \\
& & & 3 & 43.33 & 24.19 & .017 & 1.91 \\
\hline
\end{tabular}

Table 8: Comparison of N-W scores across groups

\begin{tabular}{lcccccc}
\hline \multicolumn{1}{c}{$N$-W scores } & \multicolumn{2}{c}{ dyslexia } & \multicolumn{2}{c}{ control } & & \\
\hline program & mean & SD & mean & SD & $\mathrm{t}(26)$ & $\mathrm{p}$ \\
\hline 1 - story & -0.07 & 0.09 & -0.18 & 0.09 & 1.44 & .162 \\
1 - execution & -0.01 & 0.07 & -0.04 & 0.08 & 0.96 & .346 \\
2 - story & -0.15 & 0.06 & -0.07 & 0.10 & $-2.50(22)$ & .021 \\
2 - execution & -0.15 & -0.06 & -0.07 & 0.10 & $2.50(22)$ & .021 \\
3 - story & -0.10 & 0.07 & -0.10 & 0.11 & 0.142 & .889 \\
3 - execution & 0.00 & 0.09 & 0.00 & 0.08 & -0.35 & .737 \\
\hline
\end{tabular}

necessary weighting the score according to the "distance" between the mismatched pairs. In our case, we set all such pairs to $-2\left(\mathrm{~S}_{i j}=\right.$ $2, i \neq j)$.

The second parameter is the gap penalty. The Needleman-Wunsch algorithm will insert a gap into the strings under comparison in order to maximize the alignment. Since this represents an imperfect match, a score is associated with gap insertion and can be configured as necessary. In our case, the gap penalty was set at -1 . A negative value favours alignment of line numbers in the gaze comparison and discourages gaps. With these parameters, a perfect string match gave a N-W score of 1 and a mismatch on every string position scored -1 .

For each participant, gaze sequences were formatted as csv files and processed in MATLAB using the ScanMatch library [14]. This gave two N-W scores for each participant per program, one for story order and the other for execution order. Using a two-tailed independent samples t-test for equality of means at a significance level p of 0.05 , we have results as shown in Table 8 .

For program 2 we observed a significant difference in the pattern match scores, with the dyslexia group showing a greater departure from the execution order of the program. Note that for program 2 execution order is the same as story order, i.e. program 2 has no loop or iteration constructs. We observe that the dyslexia group showed a greater (and arguably unnecessary) non-linear reading behaviour - unnecessary in the sense that a linear reading of the code is ideally sufficient to understand its function. This is consistent with Table 3 where program 2 exhibits the greatest non-linearity of the three programs and the one with the greatest difference between the two groups.

\section{DISCUSSION}

Hypothesis 1 predicted that programmers with dyslexia would have shorter saccades than the control group when reading program code. 
The analysis presented here would suggest that, while there is a pattern for the dyslexia group to have shorter saccade values, the difference is not statistically significant. One possible explanation for rejection of the hypothesis is that reading code is sufficiently different from reading natural text [12], such that the deficiencies at work when reading natural text do not carry over to code reading. For example, one accepted reason why dyslexic readers of natural text exhibit shorter saccades is the phonological deficit in processing exception words (such as "whole" or "receipt"), especially when these are new words, leading to multiple saccades within a word. Though new words (such as variable names) are a common feature when reading (someone else's) program code, it could be that the predominance of programming language key words, which will be familiar to the programmer if they have previous experience in that language, attenuates this effect. The important outcome is not that saccade length per se is broadly the same for the two programmer groups but that, as an indicator of a difference in reading behaviour for dyslexic readers, it is not readily apparent when reading code.

Hypothesis 2 predicted that, for programmers with dyslexia, the proportion of all saccades which are linear saccades is less than the proportion associated with the control group, reflecting a more "uncertain" gaze pattern to compensate for potential reading deficiencies. Treating gaze across the program as a whole, the data did not support the hypothesis, though it did demonstrate a tendency in this direction, with less linearity on the part of the dyslexia group. The linearity figures are broadly in keeping with the study in [6] where approximately $40 \%$ of code reading gaze behaviour is non-linear. This non-linearity is a necessary and inherent part of program comprehension for any programmer. It could be that the degree of non-linear reading necessary in program comprehension is in keeping with the non-linearity which is intrinsic to the text reading behaviour of a programmer with dyslexia.

Limited, though statistically significant, differences were exhibited at the line level in program 2 lines 3 and 9, with the dyslexia group exhibiting less linearity in both cases. Why these lines should be so is not clear. Comparing them, line 3 is the signature of the method, containing only Java keywords, line 9 on the other hand contains operators and operands and only one keyword.

When testing hypothesis 2 , while the statistically significant data is limited, the tendency points to less linearity in the gaze of the programmer with dyslexia. Previous work in computing has suggested that programmers with dyslexia might have processing advantages when developing software, arising from an enhanced spatial awareness and visual learning style [8]. There is debate and uncertainty in the wider dyslexia literature as to whether such a link exists. There is some evidence of a correlation with the presence of dyslexia and efficiencies in the holistic visualization of complex figures [15]. It remains an open question whether the visual properties of program code, especially in the context of software maintenance, enable it to be considered as a complex visual artefact processed in this way and whether the meaning conveyed by the structural layout of code, through indentation and special symbols, could lead to efficiencies in reading behaviour for the programmer with dyslexia not available when reading natural text. The complexity of addressing this question is highlighted by Floyd et al.'s findings [12] that, on the one hand, the neural representations associated with reading code are distinct from those associated with reading natural text and, on the other hand, for the particular case of the expert programmer, the neural representations of program code and natural language tend to be more similar.

As well as considering the visual properties of a program, there are many questions relating to the effect on the programmer with dyslexia of features such as program length, page scrolling and tab switching within the IDE. To the extent that such features introduce gaze behaviours which are a departure from linear reading, as well as the concurrent execution of keystrokes and mouse gestures, there is much scope for further study on how programmers with dyslexia, and programmers with neurodiverse conditions in general, perform in these more complex programming tasks.

One observation in the dyslexia literature is the detrimental effect of poor spacing on reading performance [16]. Poor spacing or "crowding" can arise in programming for a number of reasons, such as poor layout, overuse of comments or the inherent complexity of some code constructs. Code complexity can be understood in a number of ways [25] and similar to crowding in natural text, code complexity can have a detrimental effect on comprehension performance. With regard to the programmer with dyslexia, work is underway to explore the hypothesis that programmers with dyslexia are disproportionately affected by crowding in program code [20].

As shown in [6], the Needleman-Wunsch algorithm provides a useful mechanism for capturing linearity of code reading. When used in our analysis, it yielded a mixed insight. For story order, the dyslexia group showed a greater alignment for program 1, less alignment for program 2 and similar alignment for program 3 . For execution order it was a similar pattern. The only significant difference was in program 2 . This program has a single control flow path and yet the dyslexia group showed a greater departure from the execution order compared with the control group. Is it the case that a programmer with dyslexia is conditioned to approach code comprehension in a more generally non-linear way, again aligning with possible strengths in visual-spatial processing? It is also the case that, because of its single control flow path, this program has the least structural clues as to its purpose and is the most dense with respect to white space and indentation.

\section{CONCLUSION}

The analysis described in this paper extends previous work reported in [21] investigating the eye gaze behaviour of programmers with dyslexia. In this paper the focus is upon saccade data and what it can tell us about the linearity of the programmer's gaze when comprehending program code, in particular if there are differences between linearity of gaze for a programmer with dyslexia and a programmer who does not have dyslexia. In statistical terms, our findings are consistent with earlier work in that there are no clear differences between the reading style of the programmer with dyslexia compared to the programmer without dyslexia.

However, the analysis here confirms the prevalence of nonlinearity in code reading (approximately 40\%), consistent with previous studies. In probing the differences between the dyslexia and control groups, while there is limited significant difference, the analysis does open the question as to whether, for the programmer with dyslexia, a different visual-spatial approach to code comprehension 
is at work, aligning with possible strengths in their performance in holistic visualization tasks.

\section{REFERENCES}

[1] 2019. EMIP '19: Proceedings of the 6th International Workshop on Eye Movements in Programming. IEEE Press, Piscataway, NJ, USA.

[2] Hala Annabi, Karthika Sundaresan, and Annuska Zolyomi. 2017. It‘s not just about attention to details: Redefining the talents autistic software developers bring to software development. (2017). https://doi.org/10.24251/HICSS.2017.665

[3] Roman Bednarik, Teresa Busjahn, and Carsten Schulte. 2014. Eye movements in programming education: Analyzing the expert's gaze. In Proc. 1st Int. Workshop. $16-19$.

[4] Stéphanie Bellocchi, Mathilde Muneaux, Mireille Bastien-Toniazzo, and Stéphanie Ducrot. 2013. I can read it in your eyes: What eye movements tell us about visuo-attentional processes in developmental dyslexia. Research in developmental disabilities 34, 1 (2013), 452-460.

[5] Alan F. Blackwell, Marian Petre, and Luke Church. 2019. Fifty years of the psychology of programming. International fournal of Human-Computer Studies 131 (2019), 52 - 63. https://doi.org/10.1016/j.ijhcs.2019.06.009 50 years of the International Journal of Human-Computer Studies. Reflections on the past, present and future of human-centred technologies.

[6] Teresa Busjahn, Roman Bednarik, Andrew Begel, Martha Crosby, James H Paterson, Carsten Schulte, Bonita Sharif, and Sascha Tamm. 2015. Eye movements in code reading: Relaxing the linear order. In 2015 IEEE 23rd International Conference on Program Comprehension. IEEE, 255-265.

[7] Teresa Busjahn, Carsten Schulte, Bonita Sharif, Andrew Begel, Michael Hansen, Roman Bednarik, Paul Orlov, Petri Ihantola, Galina Shchekotova, Maria Antropova, et al. 2014. Eye tracking in computing education. In Proceedings of the tenth annual conference on International computing education research ACM, 3-10.

[8] Peter Coppin. 2008. Developing drawing and visual thinking strategies to enhance computer programming for people with dyslexia. In 2008 IEEE Symposium on Visual Languages and Human-Centric Computing. IEEE, 266-267.

[9] Filipe Cristino, Sebastiaan Mathôt, Jan Theeuwes, and Iain D Gilchrist. 2010. ScanMatch: A novel method for comparing fixation sequences. Behavior research methods 42, 3 (2010), 692-700.

[10] Nicholas Sheep Dalton. 2013. Neurodiversity \& HCI. In CHI '13 Extended Abstracts on Human Factors in Computing Systems (CHI EA '13). ACM, New York, NY, USA 2295-2304. https://doi.org/10.1145/2468356.2468752

[11] Maria De Luca, Marta Borrelli, Anna Judica, Donatella Spinelli, and Pierluigi Zoccolotti. 2002. Reading words and pseudowords: An eye movement study of developmental dyslexia. Brain and language 80, 3 (2002), 617-626.

[12] Benjamin Floyd, Tyler Santander, and Westley Weimer. 2017. Decoding the representation of code in the brain: An fMRI study of code review and expertise In 2017 IEEE/ACM 39th International Conference on Software Engineering (ICSE) IEEE, 175-186.

[13] Naama Friedmann and Max Coltheart. 2018. 35 .Types of developmental dyslexia Handbook of Communication Disorders (2018), 721-752. https://doi.org/10.1515/ 9781614514909-036

[14] Ian Gilchrist. 2010. ScanMatch Toolbox. https://seis.bristol.ac.uk/ psidg/ ScanMatch/. [Online: accessed 11-February-2020].

[15] Jeffrey W Gilger, Kristina Allen, and Anabel Castillo. 2016. Reading disability and enhanced dynamic spatial reasoning: A review of the literature. Brain and Cognition 105 (2016), 55-65.

[16] Simone Gori and Andrea Facoetti. 2015. How the visual aspects can be crucial in reading acquisition: The intriguing case of crowding and developmental dyslexia. Journal of vision 15, 1 (2015), 8-8.

[17] Stefan Hawelka, Benjamin Gagl, and Heinz Wimmer. 2010. A dual-route perspective on eye movements of dyslexic readers. Cognition 115, 3 (2010), 367-379.

[18] Kenneth Holmqvist, Marcus Nyström, Richard Andersson, Richard Dewhurst, Halszka Jarodzka, and Joost Van de Weijer. 2011. Eye tracking: A comprehensive guide to methods and measures. OUP Oxford.

[19] Ahmad Jbara and Dror G Feitelson. 2017. How programmers read regular code: a controlled experiment using eye tracking. Empirical software engineering 22, 3 (2017), 1440-1477.

[20] Ian McChesney and Raymond Bond. 2018. Gaze behaviour in computer programmers with dyslexia: considerations regarding code style, layout and crowding. In Proceedings of the Workshop on Eye Movements in Programming. 1-5. https://doi.org/10.1145/3216723.3216724

[21] Ian McChesney and Raymond Bond. 2019. Eye tracking analysis of computer program comprehension in programmers with dyslexia. Empirical Software Engineering 24, 3 (2019), 1109-1154.

[22] Meredith Ringel Morris, Andrew Begel, and Ben Wiedermann. 2015. Understanding the challenges faced by neurodiverse software engineering employees: Towards a more inclusive and productive technical workforce. In Proceedings of the 17th International ACM SIGACCESS Conference on Computers \& Accessibility.
ACM, 173-184.

[23] Unaizah Obaidellah, Mohammed Al Haek, and Peter C-H Cheng. 2018. A survey on the usage of eye-tracking in computer programming. ACM Computing Surveys (CSUR) 51, 1 (2018), 5.

[24] Erik D Reichle and Heather Sheridan. 2015. EZ Reader: An overview of the model and two recent applications. Oxford handbook of reading (2015), 277-292.

[25] Juergen Rilling and Tuomas Klemola. 2003. Identifying comprehension bottlenecks using program slicing and cognitive complexity metrics. In 11th IEEE International Workshop on Program Comprehension, 2003. IEEE, 115-124.

[26] Matthew H Schneps, L Todd Rose, and Kurt W Fischer. 2007. Visual learning and the brain: Implications for dyslexia. Mind, Brain, and Education 1, 3 (2007), 128-139.

[27] Zohreh Sharafi, Timothy Shaffer, Bonita Sharif, and Yann-Gaël Guéhéneuc. 2015. Eye-tracking metrics in software engineering. In 2015 Asia-Pacific Software Engineering Conference (APSEC). IEEE, 96-103.

[28] Bonita Sharif, Timothy Shaffer, Jenna Wise, and Jonathan I Maletic. 2016. Tracking Developers' eyes in the IDE. IEEE Software 33, 3 (2016), 105-108. 